\title{
A Retrospective Study of Factors Affecting Pathway and Time to Diagnosis, Time to Treatment in Children with Cancer in a Single Center in South India
}

\author{
${ }^{1}$ Department of Paediatrics, Kasturba Medical College, Mangalore \\ Manipal Academy of Higher Education (MAHE), Manipal, \\ Karnataka, India \\ ${ }^{2}$ Department of Microbiology, Kasturba Medical College, Mangalore \\ Manipal Academy of Higher Education (MAHE), Manipal, \\ Karnataka, India \\ ${ }^{3}$ Department of Surgery, Kasturba Medical College, Mangalore \\ Manipal Academy of Higher Education (MAHE), Manipal, \\ Karnataka, India \\ Ind J Med Paediatr Oncol 2021;42:247-254.
}

Harsha Prasada Lashkari¹, Shobha Prasada ${ }^{2}$ Jayatheerth Joshi ${ }^{3} \quad$ Sadashiva Rao ${ }^{3}$

\author{
Address for correspondence Harsha Prasada Lashkari, MD, DCH, \\ DNB, MRCPCH, CCT (London), Division of Paediatric Haematology \\ and Oncology, Department of Paediatrics, Kasturba Medical \\ College, Mangalore Manipal Academy of Higher Education \\ (MAHE), Mangalore 575001, Karnataka, India \\ (e-mail: Harsha.pl@manipal.edu).
}

\author{
Abstract \\ Keywords \\ - childhood cancer \\ - acute lymphoblastic \\ leukemia \\ - Time to diagnosis \\ - diagnostic delay
}

Introduction The overall cure rate of childhood cancers is above $79 \%$ in the developed world, whereas in the developing world, like in India, it is around $50 \%$. It is vital to know the routes of presentation and factors affecting the presentation of childhood cancers in primary, secondary, and tertiary care to design a better survival strategy in childhood cancer.

Objective The aim of this study was to know the factors affecting the time to diagnosis and time to treatment in children with cancers in a single center in South India.

Materials and Methods It was a retrospective cohort study of children diagnosed with cancer between January 1, 2014 and December 31, 2016 at the pediatric oncology unit, KMC Hospital Mangalore, India. The patient interval, time to diagnosis, patient's family, economic background, parental education, and referral pattern were recorded, and its impact on the time taken to diagnosis was studied. The data was analyzed using SPSS 20.0 software.

Results Out of 111 children, 72 were boys (64.8\%). Fifty-one (46\%) children belonged to the less than 5-year age group. The most common cancer was acute lymphoblastic leukemia, diagnosed in 50\% (56/111) children, followed by acute myeloid leukemia in $14 / 111(12.6 \%)$, brain tumors in $9(8.1 \%)$, and neuroblastoma in $10(9 \%)$ children. The median patient interval/patient delay was 14 days (1-90 days), referral interval was 14 days (1-150 days), and overall time to diagnosis was 41 days (1-194 days). The first contact was the pediatrician in $86 / 111$ (77.4\%). Sixty-four percent $(71 / 111)$
DOI https://doi.org/ $10.1055 / \mathrm{s}-0041-1732853$ ISSN 0971-5851
(C) 2021. Indian Society of Medical and Paediatric Oncology. This is an open access article published by Thieme under the terms of the Creative Commons Attribution-NonDerivative-NonCommercial-License, permitting copying and reproduction so long as the original work is given appropriate credit. Contents may not be used for commercial purposes, or adapted, remixed, transformed or built upon. (https://creativecommons.org/licenses/by-nc-nd/4.0/). Thieme Medical and Scientific Publishers Private Ltd. A-12, Second Floor, Sector -2, NOIDA -201301, India 
referral came from a secondary care hospital, and the remaining from the outpatient clinics. There was no difference in sex and patient interval $(p=0.278)$ and overall time to diagnosis $(p=0.4169)$, age $(p=0.041)$, mother's education $(p=0.034)$, and type of cancer ( $p=0.013$ ) were three critical factors that determined the time to diagnosis. Conclusion Majority of the children diagnosed with cancer presented via referral from pediatricians. An equal number of them were referred to as routine and emergency patients. Age, mother's education, and type of cancers were the crucial factors associated with the overall time taken to diagnosis.

\section{Introduction}

Cancer is an important cause of death in both children and adults. Around $70 \%$ of these cancer-related deaths happen in low- and middle-income countries such as India. ${ }^{1}$ As per the Population-Based Cancer Registry (2012-2014) report, childhood cancer accounts for 0.7 to $4.4 \%$ of total cancer diagnoses in India..$^{2-5}$ The reported standardized incidence rate for India varies from 38 to 124 per million children per year. ${ }^{6}$ Childhood cancer is predominantly not amenable to preventive strategies. Over the last few decades, in the developed world, the overall cure rate of childhood cancers has been above $70 \%$, whereas in India, we struggle to cross 50\%. ${ }^{6}$ India's low survival is due to various factors such as presentation in an advanced stage of cancer, delay in diagnosis, poor access to treatment, treatment abandonment, poor supportive care, and poor infection control practices. ${ }^{6,7}$ With the significant progress in the treatment and survival of childhood cancers in the developed world, the focus has shifted to early detection to reduce the treatment-related side effects, especially for those types of cancer, which carry an excellent prognosis. ${ }^{8,9}$

In the initial part of cancer, signs and symptoms could mimic the common childhood illnesses, which may cause a delay in diagnosis. ${ }^{10,11}$ It is essential to know the patient's pathway from the onset of symptoms to final diagnosis, to avoid a delay in diagnosis, which plays an essential role in better survival strategy. ${ }^{10-12}$ There are many studies published on delay in diagnosis in children with cancer in developed countries. ${ }^{9}{ }^{11,13-15}$ However, only a few articles have been published on this subject from developing countries. ${ }^{16-19}$ We do not have any study from the author's region in India done on routes of presentation of childhood cancer, time to diagnosis (TTD), and time to start treatment.

\section{Materials and Methods}

It was a retrospective cohort study of 111 children diagnosed with cancer between the January 1, 2014 and December 31, 2016 (3 years) at a teaching hospital, Mangalore, India. All children aged between 0 and 18 years diagnosed with cancer during the study period were included in the study, while in those where we could not retrieve the required information were excluded. It is a private tertiary care teaching hospital with a dedicated pediatric hematology/oncology unit, which is the only center in Dakshina Kannada district. The majority of patients are from below poverty line (BPL) as per the Government of India criteria and remaining from lower-middle socioeconomic status. The catchment area has been quite large, around $400 \mathrm{~km}$ but an average of $100 \mathrm{~km}$. All those from BPL background received treatment under government scheme (Suvarna Arogya trust), ${ }^{20}$ and others received treatment by obtaining funds from various nongovernment organizations or self-funds. The details of age, sex, address, distance from the treatment center, rural/urban area, parents, education status, parents economic status, number of siblings, duration of symptoms before seeing a doctor (symptom interval), time to presentation to a pediatric oncologist, TTD (diagnosis interval) and time to initiate treatment after confirming the diagnosis, and type of cancer were captured. An interview with all parents conducted in the year 2017 to collect the information not available from the case notes. The primary outcome was to know the factors affecting the overall time taken to diagnosis. The secondary outcomes were to know the patient interval, referral interval, and time taken to treatment.

\section{Definitions (-Fig. 1)}

Patient interval $^{21,22}$ : It is defined as the time between symptom onset and first clinical presentation. It is also called patient delay, duration of symptoms before the presentation

Referral interval: It is defined as the time from first seen by a pediatrician or a general practitioner to presentation to a pediatric oncologist.

Diagnostic interval: It is referred to the period from direct engagement with a healthcare professional to a definitive diagnosis (also referred to as doctor, physician, or healthcare delay)

Overall TTD/symptom interval: It is defined as the time from initial symptoms to diagnosis at our pediatric oncology unit. The time between symptom recognition and a definitive diagnosis was described as TTD.

Treatment interval (time to initiate treatment): It is defined as time taken to commence treatment from the diagnosis.

Time to treatment: It is defined as time from initial symptoms to commencement of treatment; whether surgery or chemotherapy. 


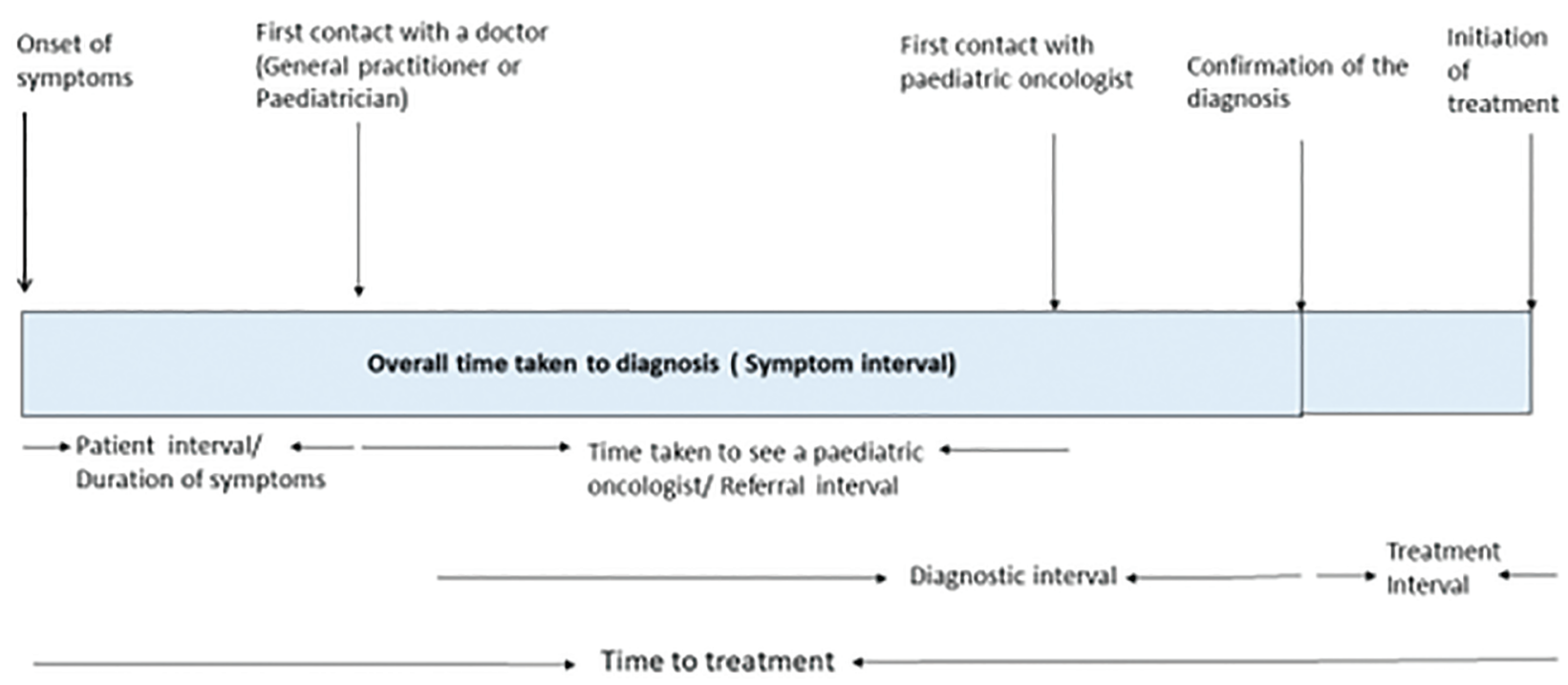

Fig. 1 Schema of patient interval, referral interval, diagnostic interval, and symptom interval.

The date of onset of symptoms and first contact with a healthcare professional were based on recall by the parents (parents interview), while the date of the first visit to the pediatric oncology center, date of confirmation of diagnosis, and date of initiation of treatment were recorded from the hospital case records.

\section{Statistical Analysis}

Univariate analysis was used to know the factors associated with total delays and assess each factor's impact separately on the patient interval, and total time taken to diagnosis. We also used it to determine correlations between possible contributing variables and time taken to diagnosis. For comparison of two groups of categorical variables, independent $t$-tests were used (as in sex variable). To compare more than two categorical variables (as in age groups, family size, father and mother's education, residence, and family income variables) one-way analysis of variance (ANOVA) was used. We used Tamhane's T2 multiple comparison tests to compare total intervals between different groups between other group pairs after ANOVA. In multivariable analysis, statistically significant variables in the ANOVA were included using a linear regression model for continuous data to estimate independent factors associated with patient interval and time taken to diagnosis. All analyses were performed using SPSS software (IBM SPSS Statistics for Windows, version 20.0, Armonk, New York, United States: IBM Corp). A $p$-value $\leq 0.05$ was considered statistically significant at a $95 \%$ confidence level. The missing data were addressed by complete case wise analysis or list wise deletion.

Ethics: The Institutional Ethics Committee of of Kasturba Medical College Hospital Mangaluru(IEC KMC MLR 09-16/245) approved this study. The procedures followed were in accordance with the ethical standards of the responsible committee on human experimentation and with the Helsinki Declaration of 1964 , as revised in 2013 . Consent has been obtained initially at the time of commencement of treatment to collect the data about the family size, socioeconomic status, parents' education, and underlying type of cancer.

\section{Results}

A total of 115 children were registered with childhood cancer during the study period. We could record the details of 111 children, while we could not collect data for remaining four children due to insufficient information in the case records ( - Table $\mathbf{1}$ ). The median duration of the patient interval, meaning onset of symptoms to the first contact of a doctor, was 14 days (95\% confidence interval [CI]: 14-19; range: 1-90 days). The median duration of diagnostic interval (from the first contact of a doctor to final diagnosis by a pediatric oncologist) was 21 days (95\% Cl: 18-31 days). For the parents and patient, the first contact was the pediatrician in $86 / 111$ (77.4\%) patients, followed by a general practitioner in eight children; a pediatric surgeon, general surgeon and general physician saw four children each. A total of 71/111 (64\%) referral came from a secondary care hospital and remaining from the outpatient clinics. Fourteen (12.6\%) children were directly admitted to the intensive care unit. The referral was an emergency in 56 (50.5\%) patients. There was no difference in sex and patient interval $(p=0.278)$, time to present to a pediatric oncologist, time taken to diagnosis $(p=0.976),(p=0.4169)$, and time to commence treatment $(p=0.688)$ on unpaired $t$-test. The number of siblings did not make any difference in the duration of various intervals $(p=0.16)$. Forty-nine children were from families with monthly income < Rs 10,000 and 58 were from family with income of Rs 25,000 to 50,000 , and rest four were from the family of income with $>$ Rs 50,000/month. Family income was an essential factor in the patient interval $(p=0.02)$. An equal number of children were from within and outside the district. Six fathers (5.4\%) and nine (8.1\%) mothers were illiterate. High school level education was completed in 70/111 (64.1\%) fathers and 76/111 (67.1\%) mothers. On ANOVA test, only the mother's education levels were significant $(p=0.038)$ in determining the time taken to presentation to a doctor (patient interval) as well as for time taken to diagnosis (-Table 1). The majority of children 70/111 (63.8\%) 


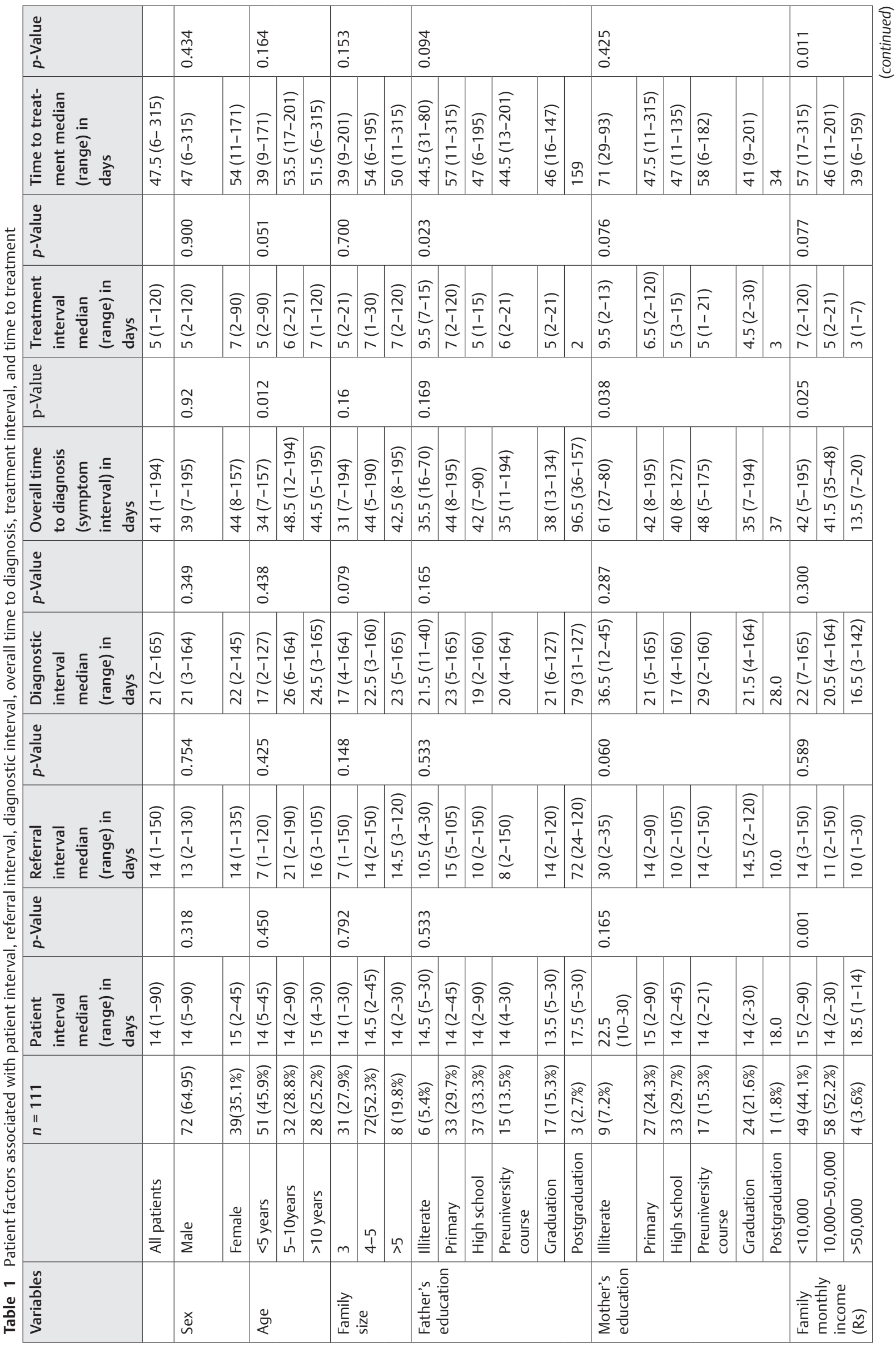




\begin{tabular}{|c|c|c|c|c|c|c|c|c|c|}
\hline \multirow{2}{*}{ 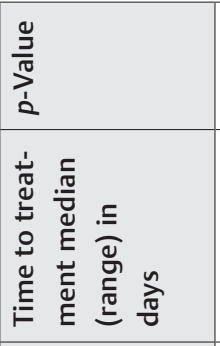 } & \multicolumn{2}{|l|}{ 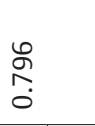 } & \multicolumn{2}{|l|}{\begin{tabular}{l}
0 \\
\multirow{6}{*}{} \\
0
\end{tabular}} & \multicolumn{2}{|l|}{\begin{tabular}{l}
$\mathscr{2}$ \\
\multirow{2}{*}{} \\
0
\end{tabular}} & \multicolumn{3}{|l|}{$\begin{array}{l}\infty \\
\tilde{O} \\
0 \\
0\end{array}$} \\
\hline & 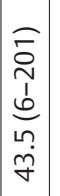 & $\begin{array}{l}\frac{1}{n} \\
\frac{m}{1} \\
\vdots \\
0 \\
0 \\
n\end{array}$ & 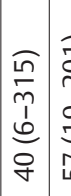 & 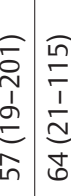 & 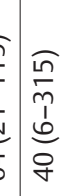 & 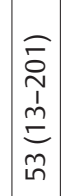 & 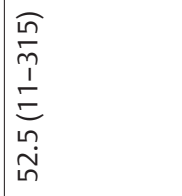 & 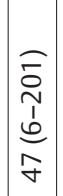 & 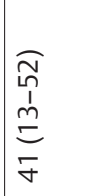 \\
\hline $\begin{array}{l}\frac{9}{3} \\
\frac{m}{10} \\
\frac{1}{2} \\
\end{array}$ & \multicolumn{2}{|l|}{ 寅 } & \multicolumn{2}{|l|}{$\begin{array}{l}m \\
0 \\
0 \\
0\end{array}$} & \multicolumn{2}{|l|}{ 竎 } & \multicolumn{3}{|l|}{$\begin{array}{l}0 \\
0 \\
0 \\
0\end{array}$} \\
\hline 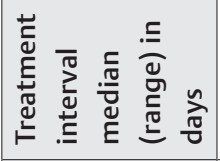 & 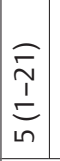 & 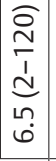 & 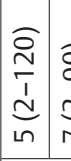 & 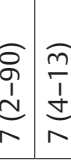 & 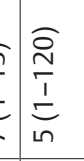 & 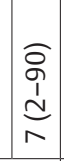 & 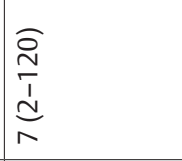 & 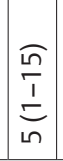 & $\begin{array}{l}\mathbb{a} \\
\stackrel{1}{d} \\
m \\
m\end{array}$ \\
\hline 墨 & \multicolumn{2}{|l|}{ ô. } & \multicolumn{2}{|l|}{$\underset{0}{0}$} & \multicolumn{2}{|l|}{$\begin{array}{l}9 \\
0 \\
0 \\
0\end{array}$} & \multicolumn{3}{|l|}{$\begin{array}{l}\tilde{\sigma} \\
\tilde{\sigma} \\
0\end{array}$} \\
\hline 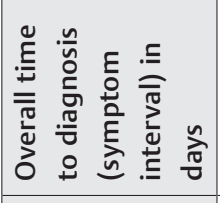 & 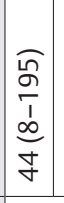 & 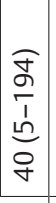 & 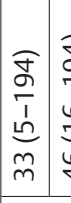 & 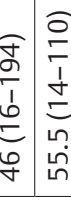 & 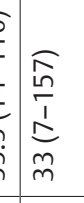 & 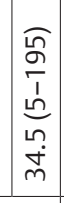 & 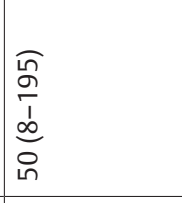 & 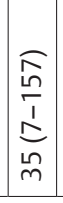 & 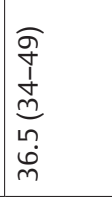 \\
\hline$\frac{9}{\frac{9}{m}}$ & \multicolumn{2}{|l|}{$\begin{array}{l}\hat{h} \\
\stackrel{0}{0} \\
0\end{array}$} & \multicolumn{2}{|l|}{\begin{tabular}{|l}
0 \\
0 \\
0 \\
0
\end{tabular}} & \multicolumn{2}{|l|}{$\frac{9}{2}$} & \multicolumn{3}{|l|}{ 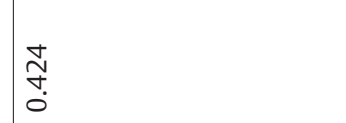 } \\
\hline 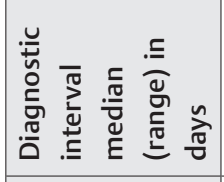 & 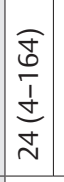 & 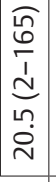 & 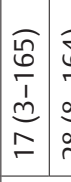 & 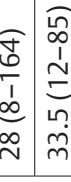 & 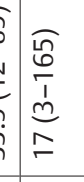 & 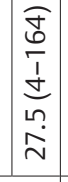 & 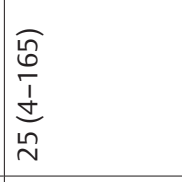 & 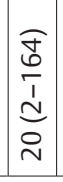 & 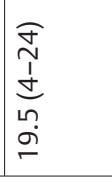 \\
\hline$\frac{0}{\frac{9}{2}}$ & \multicolumn{2}{|l|}{ 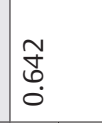 } & \multicolumn{2}{|l|}{ 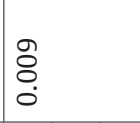 } & \multicolumn{2}{|l|}{\begin{tabular}{|l}
$\bar{\infty}$ \\
0 \\
0 \\
\end{tabular}} & \multicolumn{3}{|l|}{$\begin{array}{l}\stackrel{\infty}{ } \\
\stackrel{0}{0} \\
0\end{array}$} \\
\hline 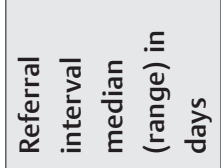 & 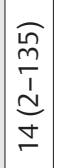 & 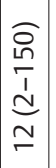 & 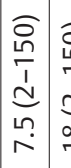 & 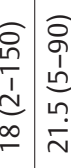 & $\hat{\imath}$ & 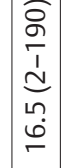 & 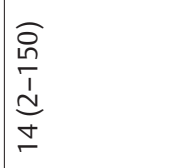 & 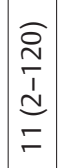 & 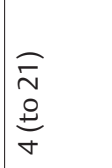 \\
\hline$\frac{9}{\frac{9}{2}}$ & \multicolumn{2}{|l|}{ 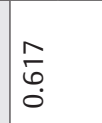 } & \multicolumn{2}{|l|}{$\begin{array}{l}\infty \\
\infty \\
0 \\
0\end{array}$} & \multicolumn{2}{|l|}{$\begin{array}{l}\stackrel{a}{2} \\
\hat{n} \\
0\end{array}$} & \multicolumn{3}{|l|}{$\begin{array}{l}\text { g) } \\
\infty \\
0 \\
0\end{array}$} \\
\hline 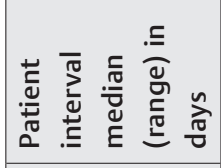 & 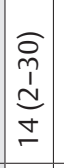 & 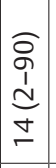 & 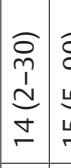 & 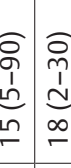 & 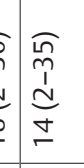 & 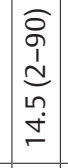 & 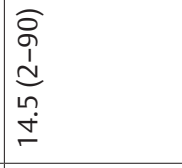 & 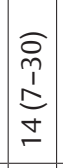 & 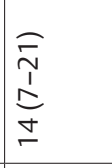 \\
\hline $\begin{array}{l}\bar{E} \\
\overline{I I} \\
=\end{array}$ & 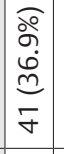 & 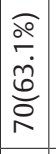 & 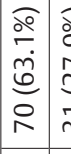 & 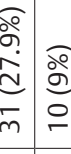 & 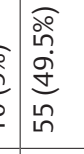 & 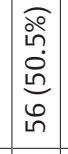 & 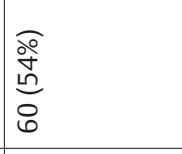 & 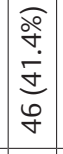 & 离 \\
\hline & $\begin{array}{l}\text { 䎡 } \\
\text { 站 } \\
\end{array}$ & 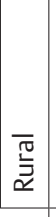 & 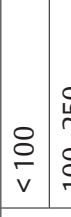 & $\begin{array}{c}0 \\
\stackrel{0}{n} \\
\vdots \\
\vdots \\
0\end{array}$ & 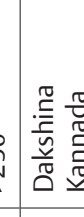 & 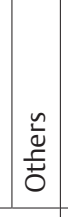 & 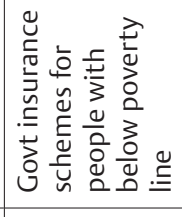 & & 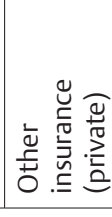 \\
\hline 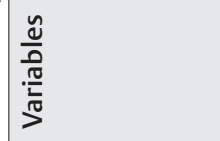 & 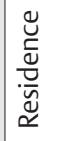 & \multicolumn{3}{|c|}{ 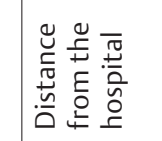 } & \multicolumn{2}{|l|}{ 蒙 } & \multicolumn{3}{|l|}{ 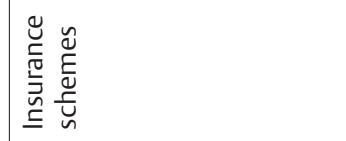 } \\
\hline
\end{tabular}


Table 2 Different types of cancers and median patient interval, referral interval, diagnostic interval, symptom interval, treatment interval, and time to treatment

\begin{tabular}{|c|c|c|c|c|c|c|c|}
\hline & $n=111$ & $\begin{array}{l}\text { Patient } \\
\text { interval } \\
\text { (days) }\end{array}$ & $\begin{array}{l}\text { Referral } \\
\text { interval } \\
\text { (days) }\end{array}$ & $\begin{array}{l}\text { Diagnostic } \\
\text { interval } \\
\text { (days) }\end{array}$ & $\begin{array}{l}\text { Overall time } \\
\text { to diagnosis } \\
\text { (symptom } \\
\text { interval) (days) }\end{array}$ & $\begin{array}{l}\text { Treatment } \\
\text { interval } \\
\text { (median in } \\
\text { days) }\end{array}$ & $\begin{array}{l}\text { Time to } \\
\text { treatment } \\
\text { (median in days) }\end{array}$ \\
\hline$p$-Value & & 0.003 & 0.075 & 0.080 & 0.001 & 0.626 & 0.3 \\
\hline $\begin{array}{l}\text { Acute lymphoblastic } \\
\text { leukemia }\end{array}$ & $56(50.4 \%)$ & $14(2-45)$ & $11(1-150)$ & $18(4-160)$ & $33(5-175)$ & $5(1-90)$ & $38(6-182)$ \\
\hline $\begin{array}{l}\text { Acute myeloid } \\
\text { leukemia }\end{array}$ & $14(12.6 \%)$ & $14(1-30)$ & $5(1-30)$ & $12.5(5-34)$ & $32(7-49)$ & $5(2-14)$ & $22(9-54)$ \\
\hline Brain tumors & $9(8.10 \%)$ & $15(5-35)$ & $23(3-120)$ & $36(6-127)$ & $63(16-157)$ & $14(2-21)$ & $82(17-159)$ \\
\hline Neuroblastoma & $10(9.00 \%)$ & $14(5-30)$ & $10.5(7-30)$ & $22(13-40)$ & $39(18-65)$ & $5(2-8)$ & $49(26-72)$ \\
\hline Lymphoma & $9(8.10 \%)$ & $\begin{array}{l}21 \\
(14-90)\end{array}$ & $30(7-150)$ & $45(17-165)$ & 70 (32-195) & $7(5-60)$ & $80(40-315)$ \\
\hline $\begin{array}{l}\text { Others (Wilms } \\
\text { tumors 4, Ewing's } \\
\text { 2, osteosarcoma } \\
\text { 2, rhabdomyosar- } \\
\text { coma 2, JMML 2, } \\
\text { retinoblastoma 1, } \\
\text { teratoma } 1 \text { ) }\end{array}$ & $13(11.7 \%)$ & $21(7-30)$ & $18(1-75)$ & $28(2-85)$ & 52 (9-99) & $9(2-15)$ & $65(29-103)$ \\
\hline
\end{tabular}

Abbreviation: JMML, juvenile myelomonocytic leukemia.

were residing in a rural area. It does not have any correlation with the TTD ( $p=0.97)$. The distance from the hospital is an essential factor in the patient interval as well as for the overall time taken to diagnose $(p=0.02)$. Sixty-four children (58\%) had insurance schemes, of which 60 (54\%) had the government insurance schemes meant for children from an economically poor background. This factor also played important role in determining the time taken for an overall diagnosis $(p=0.023)$. The most common cancer diagnosed in 50\% (56/111) patients was acute lymphoblastic leukemia (ALL), ${ }^{23}$ followed by acute myeloid leukemia (AML) in $14 / 111(12.6 \%)$ children. The brain tumors were noted in nine $(8.1 \%)$ and neuroblastoma in ten $(9 \%)$ children. The median overall time taken to diagnosis was 70 days for lymphomas (32-195 days), 63 days for brain tumors (12-157) days, and 33 days for ALL (5-175) days (-Table 2). The one-way ANOVA test on comparing the means of patient interval and diagnosis, referral interval and underlying diagnosis, diagnostic interval and diagnosis, and the overall time taken to diagnosis showed that patient interval, diagnosis interval, and the overall time taken to diagnosis were significant between the different types of cancer $(p<0.05)$. The median referral interval for ALL was 11 days. The overall time to initiate treatment after diagnosis varied between 1 and 90 days with a median of 5 days for ALL (1-90), 12 days for brain tumors (2-21), 7 days for neuroblastoma (2-8), and 7 days for lymphoma (5-60) days (- Table 2 ).

Age, mothers' education, distance from the hospital, family income, insurance schemes, and underlying diagnoses were significant for patient interval and the overall time taken to diagnosis on the ANOVA test. On multivariable analysis using a linear regression model of the above variables, age $(p=0.041)$, mothers' education $(p=0.034)$, and type of cancer $(p=0.013)$ were three critical factors that determined the time taken to diagnosis.

\section{Discussion}

Overall TTD of childhood cancer varies between cancer types. In our study, the overall time taken to diagnosis was better than the study reported by Verma and Bhattacharya. ${ }^{24}$ This is likely due to primary care pediatricians and general practitioners quickly referred these children to a pediatric oncologist. Also, most of the doctors who have referred to us were working in their establishment clinics, which could have played a role in early referral. The overall time taken to diagnose was maximum for bony sarcomas and least for acute leukemias, similar to a study published by Brasme et al. ${ }^{25}$ Earlier studies have shown that TTD depends on the cancer type, shortest for abdominal tumors ${ }^{26}$ and the longest time for brain tumors. ${ }^{27}$ The median duration of onset of symptoms to confirmation of the diagnosis in acute leukemia was 33 days. Ewing's sarcoma has one of the most prolonged intervals between onset of symptoms and diagnosis. ${ }^{22-24}$ Lethaby et al, ${ }^{11}$ in their systematic review, found out that bone tumors and brain tumors were taking a long-time average of 100 to 110 days to diagnosis. ${ }^{28}$ Stefan and Siemonsma ${ }^{29}$ published a study where an average of 20 days delay was attributed to the physician. Likewise, Araz and $\mathrm{Guler}^{30}$ also noted physician delay in the diagnosis. In our study, the median referral interval was 17.5 days, which was better than the rest of the published studies. This could happen because of the average education level of both parents and awareness of childhood cancer among the primary care doctors, mainly pediatricians.

Our study showed no correlation between the child's sex with patient interval or time taken to diagnosis. A few studies 
published from India ${ }^{24}$ showed that the female child had to wait more days to consult a doctor. Gender bias in seeking healthcare or cancer treatment has been previously reported in lower and middle-income countries, especially from India. ${ }^{6}$

A child's age was the most influencing factor in determining the patient interval and the time taken to diagnose. In our study, age played an important role in seeking medical advice at first and time taken to diagnosis, which is statistically significant. The older age children ( $>10$ years) had a longer duration of symptoms before confirmation of the diagnosis. Likewise, several studies ${ }^{16,30,31}$ reported that older the child, more the delay in seeing a doctor and for TTD. In hematological malignancies, Venkatasai et $\mathrm{a}^{18 .}$. did not find any relation with delay in the child's presentation and age.

Our study showed that a mother's education plays an essential role in the time taken to diagnose. In contrast to this, study from Bangladesh by Begum et al, ${ }^{19}$ showed no relationship with the mother's education. Instead, father education played an important role in taking these children to doctors and getting the diagnosis. A study from Israel ${ }^{31}$ reported that working mothers took more time to see a doctor, and the father's education did not affect the patient interval. This difference in findings between our study and rest could be because of family customs or male-dominated society.

There was a statistically significant correlation between family income and time to see a doctor or TTD in our study. Similar findings were noted in Venkatasai et $\mathrm{al}^{18}$ and Begum et al. ${ }^{19}$ However, Verma and Bhattacharya ${ }^{24}$ did not show any correlation with economic background. Our study, like a study by Abdelkhalek et al, ${ }^{17}$ did not reveal any correlation between the patient's places of residence, whether it was rural or urban to TTD. However, studies from the rest of India, by Venkatasai e $\mathrm{al}^{18}$ and Verma and Bhattacharya, ${ }^{24}$ have found that children from rural backgrounds take a longer time to see a doctor and end up having a longer TTD. This could be because of the high literacy rate in the author's region. Some of the patients from rural backgrounds in Venkatasai's group received alternative medicine therapy, which caused a delay in diagnosis. Also, parents from rural areas were from poor socioeconomic backgrounds, and their education level was lower.

In our study, the median time required initiation of treatment was around 5 ( 1 to 90 ) days. A majority of children were from low monthly income backgrounds, and they had to wait for the approval of the application for funding for the treatment. However, there is no influential association of TTD with better survival; TTD is a factor in reducing the morbidity associated with the condition. ${ }^{11}$

Diagnosis of cancer through an emergency presentation route is presumed to be an indicator of late diagnosis and a corresponding poor prognosis. In this study, an equal number of children presented to the emergency and the clinic. It did not have any impact on the patient interval or to symptom interval. There is a need to find out the extent of delay in diagnosis of cancer in across the country by doing prospective, multicenter study, and addressing the factors responsible for the same to improve the overall survival.

\section{Limitations}

It was a retrospective study and therefore subject to certain limitations inherent in their design. In addition, it had a relatively small sample size. The use of preexisting case records makes it difficult to obtain information on potential confounding variables. Parents and patients have recalled certain events, especially the onset of symptoms and first contact with a doctor.

\section{Conclusion}

Overall, our study showed a statistically significant association with the age, mother's education, and diagnosis with the patient interval and overall time taken to diagnosis. A prospective nationwide study may help to plan and implement the intervention programs to reduce delays.

\section{Authors' Contribution}

Harsha Prasada Lashkari conceived the idea, and designed, analyzed, and wrote the manuscript. Shobha Prasada helped to write the draft. Jayatheerth Joshi and Sadashiva Rao helped in referring the patients for the recruitment of the study. All the authors have approved the final version to be published and agree to be accountable for all aspects of the work.

\section{Funding}

None.

\section{Conflicts of Interest}

There are no conflicts of interest.

\section{Acknowledgments}

We would like to thank the families and children for their participation, Miss Madhura Kishore and Miss Shobha Naik for collecting the data, and my pediatric department colleagues for referring the cases.

\section{References}

1 WHO. Facts about Hungary. 2011. Available from: http:// www.xpatloop.com/news/who_facts_about_health_in_hungary. Accessed July 2, 2021

2 Bashar MA, Thakur JS. Incidence and pattern of childhood cancers in India: Findings from population-based cancer registries. Indian J Med Paediatr Oncol 2017;38(2):240-241

3 Bashar A. Incidence and pattern of childhood cancers in India: findings from population-based cancer registries. Indian J Cancer 2016;53(4):511-512

4 Arora RS, Alston RD, Eden TOB, et al. Cancer at ages 15-29 years: the contrasting incidence in India and England. Pediatr Blood Cancer 2012;58(1):55-60

5 Three year report of PBCR. Available from: http://ncdirindia. org/NCRP/ALL_NCRP_REPORTS/PBCR_REPORT_2012_2014/ ALL_CONTENT/Printed_Version.htm. Accessed July 2, 2021

6 Arora RS, Eden TO, Kapoor G. Epidemiology of childhood cancer in India. Indian J Cancer 2009;46(4):264-273

7 Ganguly S, Kinsey S, Bakhshi S. Childhood cancer in India. Cancer Epidemiol 2021;71(Pt B):101679

8 Reaman GH. A national approach to evaluating delays in diagnosis and treatment: potential implications for age related 
and geo-political differences in access to care and outcome in children and adolescents with cancer. Pediatr Blood Cancer 2008;51(4):447-448

9 Dang-Tan T, Trottier H, Mery LS, et al. Determinants of delays in treatment initiation in children and adolescents diagnosed with leukemia or lymphoma in Canada. Int J Cancer 2010;126(8):1936-1943

10 Rogers EK, Cannon A, Zaborowski K, Paul SP. Early recognition and management of brain tumours in children. Nurs Stand 2016;31(1):42-49

11 Lethaby CD, Picton S, Kinsey SE. Phillips R, van Laar M, Feltbower RG. A systematic review of time to diagnosis in children and young adults with cancer. Arch Dis Child 2013;98(5):349-355

12 Clarke RT, Jones CHD, Mitchell CD, Thompson MJ. 'Shouting from the roof tops': a qualitative study of how children with leukaemia are diagnosed in primary care. BMJ Open 2014;4(2):e004640

13 Roskin J, Diviney J, Nanduri V. Presentation of childhood cancers to a paediatric shared care unit. Arch Dis Child 2015;100(12):1131-1135

14 Dang-Tan T, Trottier H, Mery LS, et al. Delays in diagnosis and treatment among children and adolescents with cancer in Canada. Pediatr Blood Cancer 2008;51(4):468-474

15 Hansen RP, Olesen F, Sørensen HT, Sokolowski I, Søndergaard J. Socioeconomic patient characteristics predict delay in cancer diagnosis: a Danish cohort study. BMC Health Serv Res 2008;8:49

16 Abdelmabood S, Kandil S, Megahed A, Fouda A. Delays in diagnosis and treatment among children with cancer: Egyptian perspective. East Mediterr Health J 2017;23(6):422-429

17 Abdelkhalek E, Sherief L, Kamal N, Soliman R. Factors associated with delayed cancer diagnosis in Egyptian children. Clin Med Insights Pediatr 2014;8:39-44

18 Venkatasai JP, Srinivasamaharaj S, Sneha LM, Scott JX, Baby AK, Rajan M. Pediatric hematological malignancy: identification of issues involved in the road to diagnosis. South Asian J Cancer 2017;6(1):28-30

19 Begum M, Islam MJ, Akhtar MW, Karim S. Evaluation of delays in diagnosis and treatment of childhood malignancies in Bangladesh. South Asian J Cancer 2016;5(4):192-193

20 Sood N, Bendavid E, Mukherji A, Wagner Z, Nagpal S, Mullen P. Government health insurance for people below poverty line in
India: quasi-experimental evaluation of insurance and health outcomes. BMJ 2014;349:g5114

21 Weller D, Vedsted P, Rubin G, et al. The Aarhus statement: improving design and reporting of studies on early cancer diagnosis. Br J Cancer 2012;106(7):1262-1267

22 Cecen E, Gunes D, Mutafoglu K, Sarialioglu F, Olgun N. The time to diagnosis in childhood lymphomas and other solid tumors. Pediatr Blood Cancer 2011;57(3):392-397

23 Lashkari HP, Faheem M, Sridevi Hanaganahalli B, et al. Resource limited centres can deliver treatment for children with acute lymphoblastic leukaemia with risk-stratified minimal residual disease based UKALL 2003 protocol with no modification and a good outcome. Expert Rev Hematol 2020;13(10):1143-1151

24 Verma N, Bhattacharya S. Time to diagnosis and treatment of childhood cancer. Indian J Pediatr 2020;87(8):641-643

25 Brasme JF, Chalumeau M, Oberlin O, Valteau-Couanet D, Gaspar N. Time to diagnosis of Ewing tumors in children and adolescents is not associated with metastasis or survival: a prospective multicenter study of 436 patients. J Clin Oncol 2014;32(18):1935-1940

26 Saha V, Love S, Eden T, Micallef-Eynaud P, MacKinlay G. Determinants of symptom interval in childhood cancer. Arch Dis Child 1993;68(6):771-774

27 Mehta V, Chapman A, McNeely PD, Walling S, Howes WJ. Latency between symptom onset and diagnosis of pediatric brain tumors: an Eastern Canadian geographic study. Neurosurgery 2002;51(2):365-372, discussion 372-373

28 Shanmugavadivel D, Liu JF, Murphy L, Wilne S, Walker D. Accelerating diagnosis for childhood brain tumours: An analysis of the HeadSmart UK population data. Arch Dis Child. 2020. doi:10.1136/archdischild-2018-315962

29 Stefan DC, Siemonsma F. Delay and causes of delay in the diagnosis of childhood cancer in Africa. Pediatr Blood Cancer 2011;56(1):80-85

30 Araz NC, Guler E. Delays in diagnosis of childhood cancer in southeastern Turkey and the associated factors. Pediatr Hematol Oncol 2015;32(2):153-163

31 Haimi M, Peretz Nahum M, Ben Arush MW. Delay in diagnosis of children with cancer: a retrospective study of 315 children. Pediatr Hematol Oncol 2004;21(1):37-48 